\title{
Resolving Nonstationary Spectral Information in Wind Speed Time Series Using the Hilbert-Huang Transform
}

\author{
Vincent, Claire Louise; Giebel, Gregor; Pinson, Pierre; Madsen, Henrik
}

Published in:

Journal of Applied Meteorology and Climatology

Link to article, DOI:

10.1175/2009JAMC2058.1

Publication date:

2010

Document Version

Early version, also known as pre-print

Link back to DTU Orbit

Citation (APA):

Vincent, C. L., Giebel, G., Pinson, P., \& Madsen, H. (2010). Resolving Nonstationary Spectral Information in Wind Speed Time Series Using the Hilbert-Huang Transform. Journal of Applied Meteorology and Climatology, 49(2), 253-267. https://doi.org/10.1175/2009JAMC2058.1

\section{General rights}

Copyright and moral rights for the publications made accessible in the public portal are retained by the authors and/or other copyright owners and it is a condition of accessing publications that users recognise and abide by the legal requirements associated with these rights.

- Users may download and print one copy of any publication from the public portal for the purpose of private study or research.

- You may not further distribute the material or use it for any profit-making activity or commercial gain

- You may freely distribute the URL identifying the publication in the public portal 


\title{
Resolving Non-Stationary Spectral Information
} in Wind Speed Time Series using the

\section{Hilbert-Huang Transform}

\author{
Claire Vincent * \\ Ris $\varnothing$ National Laboratory For Sustainable Energy \\ Technical University of Denmark, Roskilde, Denmark \\ GREGor GieBEL \\ Risø NAtional Laboratory FOr Sustainable EnERgy \\ Technical University of Denmark, Roskilde, Denmark \\ Pierre Pinson and Henrik Madsen \\ TEChNicAl University of DenMark, \\ Dpt. of Informatics and Mathematical Modeling, Lyngby, Denmark
}

${ }^{*}$ Corresponding author address: Claire Vincent, Risø-DTU National Laboratory for Sustainable Energy,

Frederiksborgvej 399, PO Box 49, Roskilde DK-4000.

E-mail: clav@risoe.dtu.dk 


\begin{abstract}
This work is motivated by the observation that large amplitude wind fluctuations on temporal scales of 1 to 10 hours present challenges for the power management of large offshore wind farms. Wind fluctuations on these scales are analyzed at a meteorological measurement mast in the Danish North Sea using a 4 year time series of 10 minute wind speed observations.

An adaptive spectral analysis method called the Hilbert-Huang transform is chosen for the analysis, because the non-stationarity of time series of wind speed observations means that they are not well described by a global spectral analysis method such as the Fourier transform. The Hilbert-Huang transform is a local method based on a non-parametric and empirical decomposition of the data followed by calculation of instantaneous amplitudes and frequencies using the Hilbert transform.
\end{abstract}

The Hilbert-Huang transformed 4-year time series is averaged and summarized to show climatological patterns in the relationship between wind variability and time of day. Firstly, by integrating the Hilbert spectrum along the frequency axis, a scalar time series representing the total variability within a given frequency range is calculated. Secondly, by calculating average spectra conditional to time of day, the time axis of the Hilbert spectrum is "remapped" to show climatological patterns. Finally, the daily pattern in wind variability and wind speed are compared for the four seasons of the year. It is found that the most intense wind variability occurs in Autumn even though the strongest observed wind speeds occur in Winter. 


\section{Introduction}

With wind power now accounting for around 20\% of Denmark's annual electricity consumption, there are challenges in managing the variable power supply arising from fluctuating wind speeds. On land, fluctuations from individual wind farms tend to be uncorrelated due to both the spatial distribution of the turbines and the de-correlating effect of surface roughness and topography, leading to an overall smoothing in the regional power production (Focken et al. 2002; Giebel 2007). For offshore wind farms, however, the power supply is particularly susceptible to intense fluctuations due to the uniform surface conditions and the large number of turbines located within a small geographical area. For example, Akhamatov

et al. (2007) discussed power fluctuations from the 160 MW offshore wind farm at Horns Rev, near the west coast of Denmark, which are more intense than those experienced for geographically distributed turbines on land. The power output from the Horns Rev wind farm was shown to fluctuate with an amplitude of up to $100 \mathrm{MW}$ in 15-20 min. The construction of the second Horns Rev wind farm, with a capacity of more than 200 MW in close proximity to the existing turbines (as shown in figure 1) is expected to exacerbate these problems.

The aim of this work is to apply a statistical method called the Hilbert-Huang transform to describe the time evolving variability information in wind speed time series. The applicability of the method to studying the seasonal and diurnal patterns in wind variability will be demonstrated by investigating the diurnal cycle in wind variability on temporal scales of 1 to 10 hours at the Horns Rev wind farm. The problem of forecasting wind variability will not be addressed here, but it is suggested that defining a quantitative descriptor of wind variability and determining some characteristic seasonal and diurnal trends in wind 
variability are fundamental building blocks to developing statistical or physical forecasting tools. Actually, by extracting time series of variability for different temporal scales (i.e. non-overlapping ranges of frequencies), one could develop forecasting methodologies in a multivariate time series framework, for example based on Vector Auto-Regressive models as recently proposed in (Kim et al. 2008). Understanding wind variability on all scales is not only of scientific interest, but also has very practical engineering applications for modeling, simulation and forecasting of power fluctuations and wind farm dynamics. For example, in a study by Sørensen et al. (2008), knowledge of the stochastic properties of wind speed was used as an input to a model for simulating wind farm power fluctuations. In another study by Akhamatov (2007), fluctuations in 10 minute power observations from the Horns Rev wind farm were studied, and were shown to be strongly dependent on wind direction.

The complexity of analyzing time series of wind data is that it is difficult to define any time scale on which the data could be considered stationary. Sudden changes in mean wind are observed, but more importantly there are sudden changes in the type, amplitude and frequency of fluctuations (Pinson et al. 2008). There are some philosophical difficulties in choosing a time scale on which to consider the stationarity of atmospheric time series, as discussed in section 2 of this paper, but for the purposes of this study we assume that time series of 10 minute wind observations are non-stationary.

There are both physical and statistical reasons for believing that time series of wind speed observations are not simply random fluctuations about a slow trend. For example, Brown et al. (1984) showed that a statistical distribution is insufficient to describe the structures in hourly wind measurements, and Ailliot et al. (2006) showed that the movement of larger scale meteorological structures was fundamental to statistical forecasts of wind speed. In (Pinson 
and Madsen 2008), the application of adaptive Markov-switching autoregressive models to wind power production, where the "regime state" of the wind is considered as an unobserved process, implies an underlying structure to the changes in wind speed. Further, it is well known that meteorological time series contain periodicities and structure on several scales, including the climatic, synoptic, diurnal and semi-diurnal scales, and mesoscale features such as convective roll clouds or the intermittent passage of showers. It is therefore expected that the analysis of wind variability should uncover interesting trends and patterns on a number of time scales.

Analyzing variability in time series lends itself to treatment in the spectral domain. For example, the well-known Fourier transform is useful in uncovering the set of global harmonics which dominate the oscillatory behavior of the time series. However, since it is assumed that the wind speed time series are non-stationary, a local adaptive method which can capture the instantaneous fluctuations in the data without influence of global harmonics from statistically distinct parts of the time series is required. Candidate methods for such analysis are reviewed in section 4 where it is argued that due to its ability to react quickly to changes in the time series, and due to its adaptive, non-parametric decomposition, the Hilbert-Huang transform is most appropriate for the analysis of non-stationary wind speed data.

In section 2, several arguments for the non-stationarity of wind speed data are reviewed. In section 3, the data collection and site description for this study are discussed. In section 4 , three methods of adaptive spectral analysis (the wavelets transform, data-adaptive wavelets based on singular spectrum analysis, and the Hilbert-Huang transform) are reviewed, followed by a brief theoretical summary of the Hilbert-Huang transform. In sections 
5 and 6 , the application of the Hilbert-Huang transform to wind speed data is discussed and demonstrated, and concluding remarks are given in section 7 .

\section{Stationarity of wind data}

The need to explore non-parametric and/or adaptive statistical techniques for the analysis of wind data is motivated by the assumption that wind speed data is non-stationary. The definition of a stationary time series is one where all finite-dimensional distributions are invariant for changes in time (Madsen 2007). In the analysis of wind data for wind farm forecasting, we are interested in second order stationarity, which means that both the mean and the variance are time invariant. Although there are many statistical tests for stationarity, testing wind data for stationarity is problematic because the definition of stationarity depends on the time scale upon which the data is considered.

A common class of tests for first order stationarity are based on searching for unit roots in an autoregressive model, since the existence of a root outside the unit circle means that time series will wander from its mean (Dickey et al. 1984). This method may be suitable for time series which vary slowly, but it is unsuitable for time series such as wind speeds which may undergo sudden changes in both mean and variance. Methods which may be applied to data with more complex dynamics include making cross-comparisons of the time series in temporal or spectral space. For example, Von Sachs and Neumann (2000) developed a wavelet-based test for stationarity which entails dividing the time series into segments of different lengths, calculating the periodogram for each segment, and then making crosscomparisons of pairs of segments in the frequency domain. Witt et al. (1998) proposed a 
stationarity test where comparisons of both the probability density function and the spectral density function over segments of the time series are used to establish strong stationarity. In the time domain, Schreiber (1997) divided the time series into segments, and then used pairs of segments as explanatory variables for each other. These methods have the shortcoming that it is necessary to choose some time scale over which to segment the data, and although Witt et al. (1998) argues that the removal of slow components by application of a high-pass filter could alleviate this problem, it is still evident that stationarity depends on the window length. For example, a window containing single transition from stable nocturnal to daytime convective conditions will be considered differently from a longer window in which such transitions could be seen as part of a stationary periodic process.

In a further method proposed by Andreas et al. (2008) the issue of window length was navigated by explicitly defining the time scales over which to compare adjacent sections of the time series based on physical arguments. They calculated a "memory" period based on the decorrelation time for each point in the time series, and used this to determine the number of points to include in each segment. Finally, they used standard statistical tests to assess the differences in the mean and variance of adjacent segments.

The methods of Andreas et al. (2008) are of most relevance to this study because they applied their test to sonic thermometer data and water vapor density data, and showed that the measurements were non-stationary as a result of passing clouds altering the surface fluxes over land. They do not test the stationarity of wind data directly, but it is clear that non-stationarity in temperature would drive non-stationarity in wind data due to changes in thermal stability. Even though the measurement site in this study is offshore, it is expected that areas of spatial non-stationarity will be advected over the sea. 
Further to the results from Andreas et al. (2008), there are physical arguments why wind data should be non-stationary. The large scale Rossby waves which determine the sequence of high pressure ridges and low pressure troughs means that on a synoptic scale the atmosphere changes from general conditions of subsidence, stability, and clear skies, to general conditions of convective instability, precipitation and cloudiness. On micro- and meso-scales, a different set of dynamics can dominate depending on the atmospheric conditions. For example, in a stable atmosphere, processes such as inertial oscillations, low level jets and gravity waves may dominate the variance of the wind. In a thermally unstable atmosphere, convection and updrafts and downdrafts of convective cells may be of great importance in determining the variance of the wind, and in strongly sheared environment turbulence and Kelvin-Helmholtz instability may be dominating factors (Stull 1988).

\section{Site description and data preparation}

The analysis is based on measurements from a meteorological mast of height $62 \mathrm{~m}$ located to the north west of the Horns Rev wind farm in the Danish North Sea. The position of the mast, relative to the wind farm and the west coast of Denmark, is shown in figure 1. The meteorological mast is exposed to easterly sector wind which comes from the land, and westerly sector wind which comes from the North Sea. Flow from the southeasterly direction is in the wake of the wind farm. A description of the site and the meteorological measurement mast is found in (Peña and Gryning 2008).

The results presented here used a 4 year time series of wind speed observations from a cup anemometer mounted at the top of the meteorological measurement mast. The measurement 
resolution was 10 minutes, and data availability was good, with only 203 missing observations (less than $0.05 \%$ ) for the four years.

The implementation of most spectral analysis techniques requires a time series without gaps, since a missing data point influences not only that point, but introduces bias into the analysis. For data with longer or more frequent gaps, several methods for analyzing unevenly spaced time series data exist (for example, as described in (Hocke and Kämpfer 2008), where the Lomb-Scargle spectrum was estimated, and then inverse Fourier-transformed to obtain an approximation of the missing data). Since the gaps in the data analyzed here were short and infrequent, it was sufficient to simply fill them by interpolation for the short gaps, or by matching a piece of data from the time series itself for longer gaps. A benefit of the HilbertHuang analysis is that gap-affected parts of the time evolving spectrum can be removed prior to post-processing or drawing conclusions from it.

A histogram and wind rose of the $62 \mathrm{~m}$ wind speed at Horns Rev Mast 2 for the period 2000-2003 are shown in figures 2 and 3. The wind rose shows three preferred direction ranges - between 200 and 250 degrees, between 290 and 310 degrees, and around 110 degrees. The most frequent strong winds above $15 \mathrm{~m} \mathrm{~s}^{-1}$ occur in the southwest to northwest sector, while the wind speed is greatly suppressed for the north easterly sector. 


\section{The Hilbert-Huang Transform}

a. Spectral analysis of non-stationary time series

Other than the Fourier transform, several spectral analysis techniques exist and have been applied to geophysical time series. For example, Ghil et al. (2002) applied Singular Spectrum Analysis (SSA) to a time series of the Southern Oscillation Index (SOI). SSA involves splitting the time series into segments according to some choice of an embedding dimension, finding the empirical orthogonal functions (EOFs) from the segments, and projecting the time series onto the EOFs to define the Principal Components. The method is good for analyzing non-linear time series, but assumes an underlying periodicity in the data.

For example, a significant difference in statistical properties between the segments of the time series undermines the relevance of projecting the time series onto the set of globally defined EOFs.

Another adaptive spectral analysis method is wavelet analysis, where a wavelet function which can be stretched or dilated is projected onto the data to find the most important frequencies at each time step (Labat 2005). Wavelet transforms have been used effectively to study geophysical time series such as turbulence measurements (eg Barthlott et al. (2007)), and are able to capture the changing spectral behavior in non-stationary data. Disadvantages of the method are that some a priori decision must be made about the shape of the wavelet function. This problem was addressed by Yiou et al. (2000) by applying SSA to successive sections of the data defined by a moving window such that a 'data adaptive wavelet' could be constructed. Their method was effective in uncovering a change in the frequency of the SOI, but has obvious problems with the length of time series that is required to divide it firstly 
into window functions, and secondly into segments according to the embedding dimension of the SSA.

A further method in which there has been recent interest is the Hilbert-Huang transform, and its extension the normalized Hilbert-Huang transform. The Hilbert-Huang transform, which was first introduced by Huang et al. (1998), consists of an empirical filter which decomposes the data into a linear combination of a set of basis functions called Intrinsic Mode Functions (IMFs), followed by extraction of the instantaneous amplitudes and frequencies of each component using the Hilbert transform. In an extension to the Hilbert-Huang transform, Huang (2005a) proposed normalization of the IMFs prior to extraction of instantaneous frequencies. Huang et al. (1998) built on work of Boashash (1992) and Cohen (1989) and others who defined the concept of instantaneous frequency and laid down the conditions that must be satisfied if a time series is to have a meaningful instantaneous frequency that can be calculated using the Hilbert transform. Huang's major contribution was to develop a technique for decomposing a time series into a set of time evolving basis functions, each of which satisfied the conditions for calculation of a meaningful instantaneous frequency using the Hilbert Transform. The decomposition technique is fully data adaptive, and is called the 'Empirical Mode Decomposition' (EMD).

Like wavelet analysis, the Hilbert-Huang transform facilitates the calculation of an adaptive spectrum where amplitude is expressed as a function of frequency and time. Unlike wavelet analysis, the decomposition of the time series is empirical and non-parametric, and does not require any a priori decision about the choice of a suitable wavelet function. Huang et al. (1998) systematically compared it to the wavelet transform and illustrated its potential in capturing time evolving frequency information in time series including wind speeds, wave 
height and earthquake vibrations. The advantage of the Hilbert-Huang transform over SSA is that it is an entirely local method which can describe the changing statistical properties of a non-stationary time series. Disadvantages of the method are the difficulty in constructing a theoretical description of the empirical decomposition, in conclusively showing that the basis is orthogonal, and the problem of mode mixing, where oscillations of similar frequencies are split between several IMFs. These issues will be discussed below when introducing the theory of the Hilbert-Huang transform. Since wind speed time series are subject to sudden changes in statistical properties, and we are interested in expressing the time evolving nature of the oscillatory behavior of the time series, the Hilbert Huang transform is an obvious candidate for the study of wind speed fluctuations.

Since its introduction in 1998, the Hilbert-Huang transform has been successfully applied to many problems involving non-stationary time series. For example, Veltcheva and Guedes Soares (2004) and Veltcheva and Guedes Soares (2007) used the method to study abnormal ocean waves, and Peng et al. (2005) found it to be a useful strategy for analyzing vibrations generated by industrial machinery. Shen et al. (2005) used the Hilbert-Huang transform to analyze 55 year time series of air temperature and sea surface temperature data, and showed that the annual cycle, 3-7 year cycles, multidecadal cycles and a long term trend were clearly differentiated by the technique. Of close relevance to the current work was a recent study by Rao and Hsu (2008) who applied the method to a series of hydrological and meteorological time series and systematically demonstrated the differences between Fourier analysis and Hilbert-Huang analysis. 


\section{b. Theory of the Hilbert Huang transform}

The theory of the normalized Hilbert-Huang transform, and its implementation, will be reviewed briefly here. Several detailed descriptions of the method already exist, including (Huang 2005b) and (Huang and Wu 2008). As discussed above, the normalized HilbertHuang transform consists of three steps: EMD of the time series into IMFs, normalization of the IMFs and extraction of instantaneous amplitudes, and finally extraction of instantaneous frequencies from the normalized IMFs using the Hilbert transform.

The EMD begins by defining two cubic splines. One passes through all the local maxima of the data, and the other passes through all the local minima. The average of the two splines is considered the local mean of the data, and is subtracted from the original time series. The result now has zero mean, but does not necessarily form an IMF since it can still contain introduced local extrema. The processes is repeated until convergence is obtained, at which point it satisfies the conditions of being an IMF as defined by Huang et al. (1998). In practice, convergence is defined as in (Huang et al. 2003), as the point where every local maximum-minimum pair is separated by a zero crossing, and when the number of zero crossings stays constant for $S$ iterations. $S$ is termed the stoppage, and is recommended to be a small number between 3 and 8 .

When the first IMF, $x_{1}(t)$, has been calculated using the above procedure, it is subtracted from the original signal:

$$
U_{1}(t)=U(t)-x_{1}(t)
$$

$U_{1}(t)$ is the same as $U(t)$, but has had the highest frequency fluctuations filtered from it. 
The next IMF is then extracted from $U_{1}(t)$ using the same process, so that:

$$
U_{2}(t)=U_{1}(t)-x_{2}(t)
$$

When the signal, $U(t)$, has been decomposed into its constituent IMFs, $x_{i}(t)$, it may be written as:

$$
U(t)=\sum_{i=1}^{N} x_{i}(t)+\varepsilon(t)
$$

where $N$ is the number of IMFs that have been extracted from the signal and $\varepsilon(t)$ is the low frequency trend, containing zero or one extrema.

Correct treatment of end effects in the decomposition is an ongoing question, since it involves making a reasonable prediction of the spline from the last extremum to the end of the time series (Huang and $\mathrm{Wu} 2008$ ). In this work, however, the time series are very long and we are interested in average variability conditions, so the end effects do not present any significant problem.

In a recent modification to the Hilbert-Huang transform, Huang (2005a) proposes normalizing the IMFs so that their amplitude is always unity and they contain only frequency modulations. The normalized Hilbert-Huang transform avoids the potential problem that if the spectrum of oscillations within the IMF and that of their low frequency envelope overlap, then the instantaneous frequency will not be meaningful according to the Bedrosian theorem (Huang and Wu 2008). Each IMF is normalized by dividing it by the cubic spline that passes through the absolute value of all its extrema. The normalization should be repeated iteratively until all amplitudes are equal to unity, because the spline can occasionally pass through values less than the IMF. Each IMF, $x_{i}(t)$ may then be divided into a frequency modulation part, $F_{i}(t)$ and an amplitude modulation part, $A_{i}(t)$, where $F_{i}(t)$ is the 
normalized IMF with amplitude of unity, and

$$
A_{i}(t)=\frac{x_{i}(t)}{F_{i}(t)}
$$

The normalised IMFs satisfy the special property that they each contain only one frequency at each time, so that instantaneous frequencies can be calculated using the Hilbert transform. The meaning of instantaneous frequency is not immediately obvious. Conceptually, it could be regarded as the inverse of the time taken for one complete oscillation, but as argued by Huang et al. (2009), there is no reason to expect that the frequency should remain constant throughout an entire oscillation. The appropriate definition of the local frequency is as the derivative of the phase angle, where the phase angle can be found by adding some properly chosen imaginary part to the time series.

$$
f(t)=x_{i}(t)+i g(t)
$$

such that

$$
x_{i}(t)=\operatorname{Re}[f(t)] .
$$

Clearly, any function $g(t)$ satisfies Eqs. (5) and (6). In general, however, the complex signal $f(t)$ will contain both positive and negative frequencies, and the frequencies will not have physical meaning. As discussed by Cohen (1989), a breakthrough in the idea of defining an instantaneous frequency was made by Gabor (1946) when a special function $g(t)$ was used which filtered out the negative frequency components and doubled the positive frequency components. This function was equivalent to the Hilbert transform, and is given as

$$
H_{i}(t)=x_{i}(t)+i \frac{1}{\pi} \mathrm{PV} \int_{-\infty}^{+\infty} \frac{x_{i}(\tau)}{(t-\tau)} d \tau=x_{i}(t)+i h_{i}(t)
$$


where PV refers to the principal value of the integral, which must be considered because the integrand is not defined at $t=\tau$.

Mathematically, the Hilbert transform of the original data could be calculated, but the result would be erroneous to interpret because the instantaneous frequency would be a result of all the overlapping frequencies present in the original signal (Boashash 1992). The construction of the normalized IMFs ensures that each component satisfies the condition of "monocomponent signals" as suggested by Cohen (1995) and Boashash (1992), which means that the spectrum of the time varying amplitude does not overlap with the spectrum of the time varying phase. In a practical sense, each normalized IMF should contain only one frequency at any time.

By writing the real and complex parts of the Hilbert transformed signal in polar coordinates, it is seen to be function of instantaneous phase and amplitude,

$$
H_{i}(t)=a_{i}(t) e^{i \theta_{i}(t)}
$$

where in the case of the normalized IMFs, the amplitude will be nearly equal to unity. Defining the instantaneous frequency as $\omega_{i}(t)=\frac{d \theta_{i}(t)}{d t}$, the Hilbert transform may then be written as

$$
H_{i}(t)=a_{i}(t) e^{i \int \omega_{i}(t) d t}
$$

Since the instantaneous phase may be expressed as $\theta(t)=\operatorname{Im}[\ln (H(t))]$, the instantaneous frequency, defined as the time derivative of the instantaneous phase, can be written as

$$
\omega(t)=\frac{d \theta}{d t}=\operatorname{Im}\left[\frac{1}{H(t)} \frac{d H(t)}{d t}\right] .
$$


The data is discrete, so the instantaneous frequency is then approximated as

$$
\omega(t) \approx \operatorname{Im}\left[\frac{1}{H(t)} \frac{\Delta H(t)}{\Delta t}\right]
$$

After the Hilbert transform of the $N$ IMFs has been established, the original signal may be reconstructed as

$$
U(t)=\operatorname{Re}\left[\sum_{i=1}^{N} a_{i}(t) e^{i \int \omega_{i}(t) d t}\right]+\varepsilon(t) .
$$

where $\varepsilon(t)$ is the low frequency trend in the data after the first $N$ IMFs have been filtered out.

Eq. (12) shows how the signal may be decomposed into a series of IMFs, each with time varying amplitude and phase. An example of the first five IMFs of such a decomposition for a 13 day sample of wind speed observations is given in figure 4. As discussed by Sweeney-Reed and Nasuto (2007) and Wu and Huang (2009), direct physical interpretation of a single IMF is not necessarily possible, due to the problem of mode mixing, where an IMF can contain parts of fluctuations belonging to different scales. For example, part of the diurnal cycle may appear at some times in the same IMF as parts of the synoptic cycle at other times. This problem can be observed in some of the IMFs shown in figure 4. To create IMFs which have a more direct physical meaning (as well as greater uniqueness), Wu and Huang (2009) developed the Ensemble Empirical Mode Decomposition (EEMD) methodology, where white noise is added to the time series to create an ensemble of decompositions. The white noise ensures that all frequencies are present at all times in the time series, and removes the problem of mode-mixing. By averaging the ensemble of decompositions, a true decomposition is found which has greater physical meaning, but for which the components may not exactly satisfy the the conditions of being IMFs. The EEMD technique has not been pursued here, 
but it would nonetheless be an important extension to the current work, particularly if unique physical processes (such as oscillations associated with roll vortices) were to be identified in a single IMF.

An alternative to using the Hilbert transform for calculation of instantaneous frequency is to use the direct quadrature, as discussed in (Huang et al. 2009). This method takes advantange of the fact that the phase can be calculated directly as the arctangent of the frequency modulation part of the signal divided by its quadrature. Huang et al. (2009) argues that it is a more local calculation of the instantaneous frequency, and that it avoids any remaining violations of the Bedrosian theorem.

One way of combining all of the frequency and amplitude information in the set of IMFs is to bin the instantaneous amplitude information in each IMF into regularly spaced frequency bins. This can be achieved by considering each frequency bin $(\omega, \omega+\Delta \omega]$ and equating all amplitude contributions $a_{i}(t)$ from the $N$ IMFs which correspond to $\omega_{i}(t) \in(\omega, \omega+\Delta \omega]$. The sum of the total amplitude contributions in each frequency bin at each time constitute the Hilbert Spectrum, $H(\omega, t)$. Other methods for handling this step include using nonparametric regression techniques to fit a curve through the amplitudes, which also has the advantage of smoothing the spectrum (Duffy 2004). However, since in this paper the Hilbert spectrum is finally averaged or converted to a scalar time series (to which is it easy to apply smoothing), no smoothing was applied to the raw Hilbert spectrum. The Hilbert spectrum has the same units as the original data set, as it represents amplitudes of the fluctuations.

The Hilbert spectrum for a two week sample period in 2000 is shown in the lower panel of figure 5. The total amplitude of fluctuations at a given time is a combination of all the amplitude contributions at that time. The corresponding wind speed time series is shown 
in the upper panel. It is seen that the episodes of intense wind variability are reflected as darker spots or lines on the Hilbert spectrum. The slow variability, such as that on days 6-8, with period of around 24 hours, falls outside the frequency range of the spectrum, and the absence of high frequency variability during this time is shown by the gap in the Hilbert spectrum.

\section{Applications of the Hilbert-Huang transform to the analysis of wind speed data}

The Hilbert spectrum as shown in figure 5 is a useful representation of the time evolving spectral information in the data. Since this study is focused on long time series of wind speed data, where the frequencies of interest are those at the high frequency end of the spectrum shown, it is important to be able to summarize the Hilbert spectrum to uncover the trends in the data. In this regard, the Hilbert-Huang transform has great flexibility. It can be averaged along the time axis to create a marginal spectrum, summed over the frequency axis to create a scalar time series of variability, or can be conditionally averaged on the time axis. All of these strategies can be employed, and each one brings out a different aspect of the trends and patterns in the data that may not otherwise be obvious.

Averaging the time evolving spectrum along the time axis,

$$
H(\omega)=\frac{1}{L} \sum_{t=1}^{L} H(\omega, t),
$$

is useful firstly because it is a relationship in the same form as a Fourier spectrum (that is, a one dimensional relationship between frequency and amplitude), and as such it can be used 
to show the difference between the Hilbert-Huang analysis and the Fourier analysis. Several studies have made comparisons of the Fourier spectrum and the marginal Hilbert spectrum, and shown that the methods produce similar results in cases of stationary or near-stationary time series, but that the spectra can be significantly different in the case of non-stationary or non-linear data (for example, in the meteorological and hydrological time series studied by Rao and Hsu (2008)).

Although the marginal Hilbert spectrum and the Fourier spectrum are both expressions of amplitude as a function of frequency and can be plotted on the same axis with the same units, they are not simply two different ways of calculating the same spectrum. As such, they are not expected to be identical. The Fourier transform is a decomposition into a family of stationary sine and cosine functions, while the Hilbert-Huang transform is a decomposition into a family of amplitude modulated and frequency modulated components. Therefore, the Hilbert-Huang transform is a local method, and will show a different frequency response for the non-stationary oscillation components in the data. With regard to wind speed, this may be of particular importance for the longer wavenumber components such those driven by diurnal or synoptic timescales, as well as for shorter wavenumber components such as the onset and decay of convective conditions. The diurnal cycle is modulated by factors including time of year, cloudiness and airmass changes, so it is not expected to be well represented by a stationary oscillation of period 24 hours. Similarly, the synoptic cycle is modulated by the number and amplitude of Rossby waves, so it is also expected to have a more complex spectral representation than a single spike at two or three days.

Another approach to summarize the information in the Hilbert spectrum is to aggregate the spectrum along the frequency axis. In this way, a scalar time series of variability within 
a given frequency range can be obtained, as

$$
H(t)=\sum_{\omega=\omega_{1}}^{\omega_{2}} H(\omega, t)
$$

where $H(t)$ represents the sum of all amplitudes within the frequency range $\left[\omega_{1}, \omega_{2}\right]$. Due to the superposition of fluctuations of different phase and frequency, the signal will not always be oscillating with this total amplitude. The amplitude can be interpreted as an upper bound on the total amplitude of fluctuations if all components were in phase, and combined according to linear superposition. While the assumption of linear superposition probably does not always hold, the value of creating scalar metric of the degree of variability is clear. For example, a scalar time series can be predicted using various univariate time series modeling tools, it can be predicted using relevant explanatory variables from NWP models, and further it could be used as an indicator of an impending episode of severe variability based on analysis of model data or upstream observations.

Finally, the Hilbert spectrum can be summarized by binning and averaging the data along the time axis to create conditional spectra. This is a particularly interesting application of the method, since it permits calculation of spectra for points which do not lie consecutively in the time series. Further, the fast adaptivity of the method means that the spectral information for an isolated point in the time series is likely to be reasonably free from the influence of the spectral information from nearby parts of the time series. 


\section{Results}

The four year time series of wind speed measurements was analyzed in four year-long segments of 52560 (2001-2003) or 52704 (2000) points. The temporal resolution of the data was doubled by cubic interpolation to improve the extent to which the highest frequency fluctuations were captured in the first IMF. The interpolation obviously did not add any high resolution information to the time series, but prevented the EMD from missing some of the existing information. The data was transformed in 4 separate years for computational convenience, although the EMD took less than 400 seconds for each time series of more than 52000 points on a current laptop computer.

The stopping criteria for the EMD was set at $S=3$, as recommended in Huang et al. (2003). The instantaneous amplitudes and frequencies of the IMFs were binned into frequency bins of width $5 \mathrm{e}-7 \mathrm{~Hz}$ to create the two dimensional Hilbert spectrum. After the Hilbert spectrum was created, the times corresponding to the gaps in the original time series were removed, so they were not included in the subsequent analysis.

Boxplots of the distributions of periods in each IMF, calculated as the inverse of the instantaneous frequency, are shown in figure 6 on a semilog axis. The medians of the distributions follow a straight line when plotted on a semilog axis - that is, each IMF has on average half the frequency of the previous IMF. This result is consistent with results presented in (Wu and Huang 2004) and (Flandrin et al. 2004), where it was shown that the processes of decomposing the time series into IMFs is equivalent to locally applying a filter bank of overlapping band pass filters. 
a. Comparison of the Hilbert spectrum with the Fourier spectrum: summation of the Hilbert spectrum over time

Averaging the Hilbert spectrum $H(\omega, t)$ over time yields a scalar relationship between amplitude and frequency, or the marginal Hilbert-Huang spectrum. The marginal HilbertHuang spectrum and its analogous Fourier spectrum are shown in figure 7. The marginal Hilbert-Huang spectrum was calculated as given in Eq. (13). The Fourier spectrum was calculated using the discrete fast Fourier transform and a Hanning window function with a truncation point of $L / 16$, where $L$ is the length of the time series. Applying a window function with a suitable truncation point is an effective strategy to provide a consistent estimate of the spectrum for stationary data (Madsen 2007). Even in the case of nonstationary data, the application of a window function to truncate the time series and mitigate the end effects smooths the spectrum and provides good definition for expected periodicities such as the 24 hour cycle, but it means that the spectrum is focused on the middle of the time series and the data at either end is discarded. That is, to smooth the spectrum, the data must be truncated so that not all of the time evolving statistical properties are represented equally. The truncation point here was chosen as a trade-off between representing a reasonable portion of the time series, and giving a spectrum that was suitably smooth for comparison with the Hilbert spectrum.

It is not expected that the spectra should be identical because the integrated HilbertHuang spectrum represents the time average of the instantaneous amplitude at each frequency, while the amplitudes of the Fourier series components represent the best fit of constant harmonics to the whole data set. Huang et al. (1998) argue that the Hilbert marginal 
spectrum therefore has a "totally different meaning" to the Fourier spectrum.

Despite the problems with comparing the two spectra in figure 7 (since the full time series is used in the Hilbert spectrum, while the Fourier spectrum focuses on the middle of the time series), there are some features in common between the two spectra. For example, the diurnal cycle is expected to be identified by both methods. Indeed, there is a small peak in both spectra at a period 24 hours, although because this site is off-shore the diurnal cycle is not very strong. The diurnal cycle in these wind speed measurements will be further discussed in later parts of this paper. The two spectra also show similar spectral slope.

The marginal Hilbert spectrum is smoother than the Fourier spectrum because it is actually an average of all the spectral information for a year. A further difference between the spectra is that the Fourier spectrum contains information up to the Nyquist period of $2 \Delta t$, or 20 minutes, while the Hilbert-Huang marginal spectrum requires $4 \Delta t$, or 40 minutes to resolve spectral information (Huang et al. 1998), which can be seen as a limitation of the Hilbert-Huang transform methodology.

\section{b. Time series of Hilbert spectrum derived wind variability}

The total amplitudes of variability for the two temporal ranges 1-3 hours and 3-10 hours were calculated, and results for the year 2000 are shown in figure 8. Both time series were very noisy, and were smoothed using moving average smoothing with a window length of 3 hours for the 1 to 3 hour temporal scales, and 10 hours for the 3 to 10 hour temporal scales. This is reasonable, because the time series show the variability of the Hilbert-Huang variability metric. Therefore, if an episode of variability with a period of 3 hours lasted for 
less than three hours, then it would not constitute an oscillation. As discussed in section 3, there were a small number of gaps in the original data that were filled to facilitate the analysis. In total there were 16 missing data values out of 52704 observation times in 2000 . Points in the variability time series corresponding to these missing data were removed.

The time series suggest that there are more high variability events in the Autumn and Winter months than in Spring and Summer. The highest concentration of peaks in the data occur in the months October to April. Both time series are, however, very noisy and there is no definite trend. Using a linear least squares regression, the two smoothed time series have a correlation coefficient of 0.42 to 0.51 for the 4 years, although any relationship is likely to be non-linear and would in fact be better described by a generalized correlation coefficient such as suggested in (Nielsen and Madsen 2001). Nonetheless, scatter plots of the two time series reveal no clear dependency, and this suggests that there are partly different dynamics controlling the variability on these two time scales, although it is not necessarily suggested here that the separation into 1-3 hour and 3-10 hour variability is a natural marker between two scales of atmospheric dynamics.

c. Analysis of wind variability as a function of time of day

It is well established that there is a diurnal cycle in wind speed over land (eg in (Holtslag 1984)). Coelingh et al. (1998) showed that there is a well defined diurnal cycle in the wind speed at two measurement sites near the Dutch coast, but that the diurnal cycle was almost non-existent for three offshore sites located in the Dutch North Sea. In another study, Peña and Gryning (2008) found a pronounced diurnal cycle in temperature at the Horns Rev 
Mast 2 for easterly winds, showing the influence of the land, but very little diurnal cycle in westerly winds.

Here, the existence of a diurnal cycle in wind variability is investigated, where "wind variability" refers to all periods from 1 to 10 hours. The four year, two-dimensional Hilbert spectra were averaged for each season into hourly time-of-day bins. That is, 24 conditional spectra were created, one for each time of day, with each bin containing approximately 2190 observations. The points corresponding to the missing data in the original time series were removed from the analysis. The averaged Hilbert spectra for the four seasons are shown in figure 9 . The spectra were normalized by dividing by the width of the frequency bins, and multiplied by frequency to emphasize the higher frequencies. Therefore the units of the color axis are $\mathrm{m} \mathrm{s}^{-1}$.

The 4 year scalar time series of variability for periods $1-3$ hours was also averaged into hourly bins, so that the diurnal cycle in wind variability could be directly compared with the diurnal cycle in wind speed. Note that since the mean of the data is subtracted during the EMD process, there is no implicit scaling of variability dictated by the method. Variability and wind speed as a function of time of day for the four seasons are shown in figure 10, and the two quantities are shown plotted as a scatter plot in figure 11. Figure 11 shows that the highest variability occurs in autumn, but that the highest wind speed occurs in winter. There is no strong correlation between average wind speed and average variability. There is a weak positive correlation in winter, spring and summer, and a weak negative correlation in autumn. It can therefore be seen that the diurnal cycle in wind variability does not simply follow the diurnal cycle in wind speed.

In spring, there is a late afternoon maximum in wind speed, which is followed by a 
maximum in wind variability around 3-4 hours later. The afternoon maximum in wind speed may be caused by the formation of a low level jet, in cases where warm air from day time heating over the land is advected over the cold North Sea, leading to very stable conditions (Stull 1988). Building on this hypothesis, the maximum wind variability occurs as the low level jet is diminishing in strength, and may occur as the low level jet conditions deteriorate to a more neutrally stratified nocturnal situation.

In summer, a midday minimum in wind speed is observed, which is likely to be part of the observed pattern, where a midday maximum in surface wind speed is accompanied by a minimum in wind speed just above the surface as the wind profile adjusts to the daytime heating and destabilizes (Holtslag 1984; Wieringa 1989). It is interesting to note that a minimum in wind variability lags the minimum in wind speed by around 3 hours. This may occur once the surface heating has existed for long enough for the layer of surface air to become well mixed; in this case, turbulence will be at a maximum but larger scale fluctuations may be equalized by the increasingly even distribution of momentum.

In winter, two daily peaks in both wind variability and wind speed are seen, where the two cycles are approximately in phase, while in autumn there are two daily peaks in both cycles which are approximately 6 hours out of phase. The two daily peaks are almost certainly due to the fact that we have combined data from the two flow regimes - from the land and from the sea - into the same analysis. The reasons for the relationship between the speed and variability cycles are difficult to describe from this analysis, and are fascinating areas for further analysis, through data analysis or modeling. 


\section{Conclusions}

The effectiveness of the Hilbert-Huang transform as a tool for analyzing non-stationary wind speed time series has been demonstrated using time series of wind observations from a meteorological mast near the Horns Rev wind farm. The method is particularly relevant to the analysis of wind speed time series, which are known to contain complicated statistical structure, breakpoints and periodicities.

The two dimensional Hilbert spectrum gives a clear and intuitive representation of the scales of motion that are present in wind speed time series, and of their relative weighting. The Hilbert spectrum not only shows the most variable parts of the spectrum, but gives a clue about the existence of any coherent periodicities in the time series, since there are transient sections of the spectrum that show a consistently enhanced amplitude within a given frequency range. It is not possible to define the full three dimensional structure of a wave based on a single point measurement, although a interesting development would be to use the Hilbert spectrum of several wind speed time series at nearby points to begin to develop an over all picture of the structures in the boundary layer, or to employ a method such as the bivariate EMF recently developed by Rilling et al. (2007) to study patterns in vector observations of wind speed.

The Hilbert spectrum has many applications that extend its utility past the two dimensional Hilbert spectrum. Aggregating the spectrum along the frequency axis to form time series of total variability gives rise to many analysis methodologies from time series analysis. For example the time series of wind variability lend themselves to modeling using autoregressive moving average (ARMA) or more sophisticated univariate models, or regression 
models which relate wind variability to other atmospheric observations or forecasts. For long time series, they can be binned and averaged to create a climatological picture of the types of conditions in which severe wind variability tends to occur. Further, time series of wind variability lend themselves to being used as a warning tool for wind energy applications - for example, when peaks in wind variability are identified directly either in upstream observations, or in NWP or other forecast data.

Using the Hilbert spectrum to create conditional spectra is a novel way to describe the total frequency response of the wind speed to relevant environmental parameters such as time of day. In using this method here, it was shown that the diurnal cycle in wind variability is not very strong, and can only be shown up as a slight trend in the binned and averaged time series analysis. Further, it was shown that the annual cycle in wind variability is much stronger than any diurnal cycle. It is possible to create conditional spectra based on any set of criteria, and it is expected that strong trends can be uncovered for criteria such as wind direction and time of year.

In this study, the Hilbert-Huang analysis was used to study the diurnal cycle in wind variability in the four seasons of the year. The method was effective because it permitted analysis of the spectral properties of the wind speed at different times of year. More importantly, the analysis was used to create average spectral information at each time of day, for data which do not come from consecutive parts of the time series. The results concerning the different diurnal cycles in wind speed and wind variability require further analysis for a full physical understanding, although it seems likely that the formation of a low-level jet contributed to the spring time pattern, and the formation of the midday minimum in wind speed above the surface for flow form the land contributed to the summer time pattern. 
Extensions and new applications of the Hilbert-Huang transform are still being developed, and it is likely that some of these new developments will afford new insights into the structures in wind speed data. For example, the ensemble EMD is likely to help with the physical interpretation of the IMFs, since it addresses the problem of mode mixing. Further, the direct quadrature method for calculation of instantaneous frequency is a more local method which avoids some of the short-comings of the Hilbert transform, and is likely to be a better strategy for future studies.

The methods described here are not limited to the study of the diurnal cycle, or to the analysis of wind speed. The conditional spectra and binned time series analysis could just as well be applied to other conditions of interest, such as wind direction, stability, or time of year. The analysis could also be applied to higher frequency wind speed data, where turbulence rather than low frequency variability would be the subject of study. The focus of this analysis has been on the study of climatological trends, which are useful in the process of developing forecasting models (physical or statistical) because they give a clue to the important explanatory factors which should be considered.

\section{Acknowledgments.}

Wind speed observations for this work were supplied by Vattenfall as part of the Danish Public Service Obligation (PSO) fund project "HRENSEMBLE - High Resolution ENSEMBLEs for Horns Rev" (under contract PSO-6382), which is gratefully acknowledged. The authors also acknowledge the support of the Danish PSO fund project "Mesoscale atmospheric variability and the variation of wind and production for offshore wind farms" (under 
contract PSO-7141). The authors are grateful to Alfredo Peña from Risø for detailed proofreading and assistance with figure 1 . Coordinates of the Horns Rev II wind farm were kindly supplied by Dong Energy. The insightful comments of two anonymous reviewers were much appreciated. 


\section{References}

Ailliot, P., V. Monbet, and M. Prevosto, 2006: An autoregressive model with time-varying coefficients for wind fields. Environmetrics, 17, 107-117.

Akhamatov, V., 2007: Influence of wind direction on intense power fluctuations in large offshore windfarms in the north sea. Wind Eng., 31, 59-64.

Akhamatov, V., C. Rasmussen, P. B. Eriksen, and J. Pedersen, 2007: Technical aspects of status and expected future trends for wind power in Denmark. Wind Energy, 10, 31-49.

Andreas, E. L., C. A. Geiger, G. Treviño, and K. J. Claffey, 2008: Identifying nonstationarity in turbulence series. Bound. Layer Meteorol., 127, 37-56.

Barthlott, C., P. Drobinski, C. Fesquet, T. Dubos, and C. Pietras, 2007: Long-term study of coherent structures in the atmospheric surface layer. Bound. Layer Meteorol., 125, 1-24.

Boashash, B., 1992: Estimating and interpreting the instantaneous frequency of a signal, part 1: Fundamentals. Proc. IEEE, 80, 520-538.

Brown, B., R. Katz, and A. Murphy, 1984: Time series models to simulate and forecast wind speed and wind power. J. Appl. Meteor. Climatol., 23, 1184-1195.

Coelingh, J., A. van Wijk, and A. Holtslag, 1998: Analysis of wind speed observations over the North Sea coast. J. Wind Eng. Ind. Aerodyn., 73, 125-144.

Cohen, L., 1989: Time-frequency distributions - a review. Proc. IEEE, 77, 941-981.

Cohen, L., 1995: Time Frequency Analysis. Prentice-Hall, 320 pp. 
Dickey, D. A., D. P. Hasza, and W. A. Fuller, 1984: Testing for unit roots in seasonal time series. J. Am. Stat. Assoc., 79, 355-367.

Duffy, D., 2004: The application of Hilbert-Huang transforms to meteorological datasets. $J$. Atmos. Oceanic Technol., 21, 599-611.

Flandrin, P., G. Rilling, and P. Goncalves, 2004: Empirical mode decomposition as a filter bank. IEEE Signal Process. Lett., 11, 112-114.

Focken, U., M. Lange, K. Mönnich, H.-P. Wadl, H. G. Beyer, and A. Luig, 2002: Shortterm prediction of the aggregated power output of wind farms - a statistical analysis of the reduction of the prediciton error by spatial smoothing effects. J. Wind Eng. Ind. Aerodyn., 90, 231-246.

Gabor, D., 1946: Theory of communication. J. IEE, 93, 429-457.

Ghil, M., et al., 2002: Advanced spectral methods for climatic time series. Rev. Geophys., 40, $1-41$.

Giebel, G., 2007: A variance analysis of the capacity displaced by wind energy in Europe. Wind Energy, 10, 67-79.

Hocke, K. and N. Kämpfer, 2008: Gap filling and noise reduction of unevenly sampled data by means of the lomb-scargle periodogram. Atmos. Chem. Phys., 8, 4603-4623.

Holtslag, A. A. M., 1984: Estimates of diabatic wind speed profiles from near-surface weather observations. Bound. Layer Meteorol., 29, 225-250. 
Huang, N., 2005a: Computing instantaneous frequency by normalizing Hilbert transform. U. S. Patent Office, patent number US 6,901,353.

Huang, N., 2005b: Hilbert-Huang Transform and its applications. World Scientific Publishing Company, 1-26 pp.

Huang, N., M. C. Wu, S. R. Long, S. S. P. Shen, W. Qu, P. Gloersen, and K. L. Fan, 2003: A confidence limit for the empirical mode decomposition and Hilbert spectral analysis. Proc. Roy. Soc. A., 459, 2317-2345.

Huang, N. and Z. Wu, 2008: A review on Hilbert-Huang transform: Method and its applications to geophysical studies. Rev. Geophys., 46, RG2006.

Huang, N., Z. Wu, S. Long, K. Arnold, X. Chen, and K. Blank, 2009: On instantaneous frequency. Adv. Adaptive Data Anal., 1, 177-229.

Huang, N. E., et al., 1998: The empirical mode decomposition and the Hilbert spectrum for nonlinear and non-stationary time series analysis. Proc. Roy. Soc. A., 454, 903-995.

Kim, D., S.-H. Paek, and H.-S. Oh, 2008: A Hilbert-Huang transform approach for prediction cyber-attacks. J. Korean Stat. Soc., 37, 277-283.

Labat, D., 2005: Recent advances in wavelet analysis: Part 1. a review of concepts. $J$. Hydrol., 314, 275-288.

Madsen, H., 2007: Time Series Analysis. Chapman \& Hall, 380 pp.

Nielsen, H. A. and H. Madsen, 2001: A generalization of some classical time series tools. Comput. Stat. Data Anal., 37, 13-31. 
Peng, Z. K., P. W. Tse, and F. L. Chu, 2005: An improved Hilbert-Huang transform and its application in vibration signal analysis. J. Sound and Vib., 286, 187-205.

Peña, A. and S.-E. Gryning, 2008: Charnock's roughness length model and non-dimensional wind profiles over the sea. Bound. Layer Meteorol., 128, 191-203.

Pinson, P., L. E. Christensen, H. Madsen, P. Sørensen, M. H. Donovan, and L. E. Jensen, 2008: Regime-switching modelling of the fluctuations of offshore wind generation. J. Wind Eng. Ind. Aerodyn., 96, 2327-2347.

Pinson, P. and H. Madsen, 2008: Adaptive modelling and forecasting of wind power fluctuations with Markov-switching autoregressive models. Int. J. Forecasting, submitted.

Rao, A. and E. Hsu, 2008: Hilbert-Huang Transform Analysis of Hydrological and Environmental Time Series. Springer, 372 pp.

Rilling, G., P. Flandrin, P. Goncalves, and J. M. Lilly, 2007: Bivariate empirical mode decomposition. IEEE Signal Process. Lett., 14, 936-939.

Schreiber, T., 1997: Detecting and analyzing nonstationarity in a time series using nonlinear cross predictions. Phys. Rev. Lett., 78, 843-846.

Shen, S. S. P., T. Shu, N. E. Huang, Z. Wu, G. R. North, T. R. Karl, and D. R. Easterling, 2005: HHT analysis of the nonlinear and non-stationary annual cycle of daily surface air temperature data. Hilbert-Huang transform and its applications, N. E. Huang and S. S. P. Shen, Eds., World Scientific Publishing, 187-209.

Sørensen, P., N. A. Cutululis, A. Vigueras-Rodríguez, H. Madsen, P. Pinson, L. Jensen, 
J. Hjerrild, and M. Donovan, 2008: Modelling of power fluctuations from large offshore wind farms. Wind Energy, 11, 29-43.

Stull, R. B., 1988: An Introduction to Boundary Layer Meteorology. Kluwer Academic Publishers, $666 \mathrm{pp}$.

Sweeney-Reed, C. M. and S. J. Nasuto, 2007: A novel approach to the detection of synchronisation in EEG based on empirical mode decomposition. J. Comput. Neurosci., 23, $79-111$.

Veltcheva, A. and C. Guedes Soares, 2007: Analysis of abnormal wave records by the HilbertHuang transform method. J. Atmos. Oceanic Technol., 24, 1678-1689.

Veltcheva, A. D. and C. Guedes Soares, 2004: Identification of the components of wave spectra by the Hilbert Huang transform method. Appl. Ocean Res., 26, 1-12.

Von Sachs, R. and M. H. Neumann, 2000: A wavelet-based test for stationarity. J. Time Ser. Anal., 21, 597-613.

Wieringa, J., 1989: Shapes of annual frequency distributions of wind speed obesrved on high meteorological masts. Bound. Layer Meteorol., 47, 85-110.

Witt, A., J. Kurths, and A. Pikovsky, 1998: Testing stationarity in time series. Phys. Rev. $E, 58(2), 1800-1810$.

Wu, Z. and N. Huang, 2004: A study of the characteristics of white noise using the empirical mode decomposition method. Proc. Roy. Soc. A., 460, 1597-1611. 
Wu, Z. and N. Huang, 2009: Ensemble empirical mode decomposition: A noise assisted data analysis method. Adv. Adaptive Data Anal., 1, 1-41.

Yiou, P., D. Sornette, and M. Ghil, 2000: Data-adaptive wavelets and multi-scale singularspectrum analysis. Physica D, 142, 254-290. 


\section{List of Figures}

1 Map of the west coast of Denmark, showing the location of the existing Horns Rev wind farm (Horns Rev windfarm I) and meteorological mast 2 (M2). The location of mast 2 is $55^{\circ} 31^{\prime} 8.10^{\prime \prime}$ North, $7^{\circ} 47^{\prime} 15.07^{\prime \prime}$ East. The second Horns Rev wind farm (Horns Rev windfarm II), under construction, is also shown. . 39

2 Histogram of wind speed at Horns Rev mast 2 for the years 2000-2003. . . . 40

3 Wind rose for Horns Rev mast 2 for the years 2000-2003. Angles are the direction from which the wind blows. . . . . . . . . . . . . . . . 41

4 The first five IMFs from the Empirical Mode Decomposition of a wind speed time series. The bottom panel shows the corresponding wind speed observations. 42

$5 \quad$ Hilbert Spectrum (lower panel) of 10 minute wind observations at Horns Rev Mast 2 for a two week period in February 2000. The corresponding wind speed time series is given in the upper panel. Darker contours mean higher amplitude fluctuations, and lighter contours mean smaller amplitude fluctuations. . . . 43

6 Distribution of periods for the first 10 IMFs for the years 2000-2003. The whiskers show the 90th and 10th percentiles, and the symbols *, o and + show the 5 th or 95 th, 1 st or 99 th and 0.5 th or 99.5 th percentiles respectively. 44

$7 \quad$ Smoothed Fourier spectrum (calculated using a discrete Fourier transform and a Hanning window function) and Hilbert-Huang marginal spectrum for a 1 year time series of 10 minute wind observations $\ldots \ldots \ldots \ldots$ 
8 Time series of total amplitude of fluctuations for periods 1 to 3 hours (upper panel) and 3 to 10 hours (lower panel), for the year 2000. The time series were smoothed using centered moving average smoothing with a window length of 3 hours (1 to 3 hour variability time series) and 10 hours (3 to 10 hour variability time series). . . . . . . . . . . . . . . . . . . . . . . . 46

9 Hilbert-Huang spectra, averaged for the years 2000-2003 according to time of day. Spring (top left), Summer (top right), Autumn (bottom left) and Winter

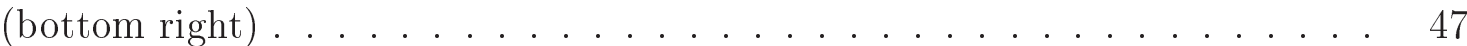

10 Upper panel: Total amplitude of variability on scales of 1-3 hours as a function of time of day, averaged for the years 2000-2003. Lower panel: Wind speed as a function of time of day, averaged for the years 2000-2003. . . . . . . . . 48

11 The same data as in figure 10, plotted as average variability on scales of 1-3 hours against average wind speed. . . . . . . . . . . . . . . . . . 49 


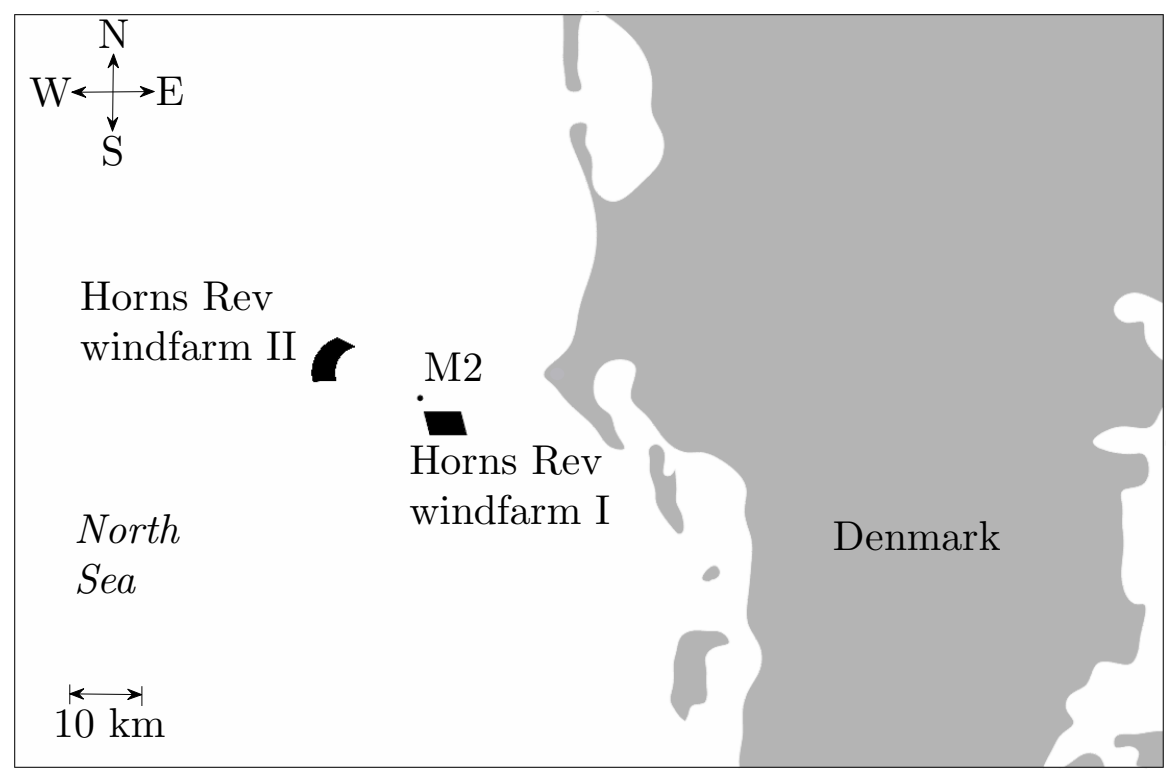

Fig. 1. Map of the west coast of Denmark, showing the location of the existing Horns Rev wind farm (Horns Rev windfarm I) and meteorological mast 2 (M2). The location of mast 2 is $55^{\circ} 31^{\prime} 8.10^{\prime \prime}$ North, $7^{\circ} 47^{\prime} 15.07^{\prime \prime}$ East. The second Horns Rev wind farm (Horns Rev windfarm II), under construction, is also shown. 


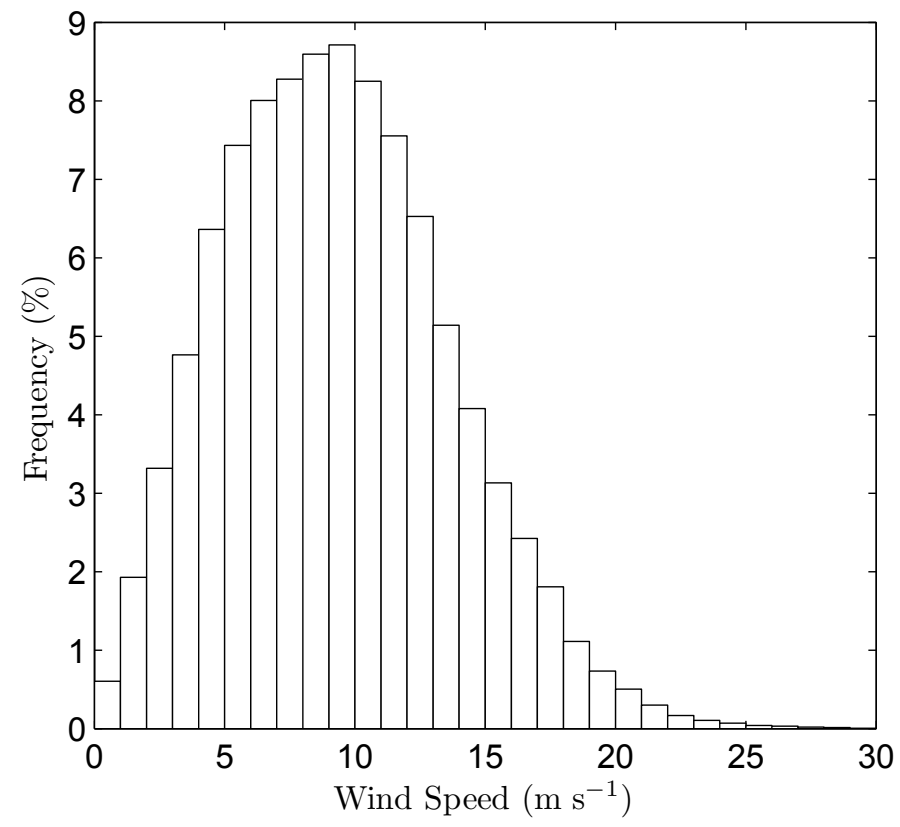

FIG. 2. Histogram of wind speed at Horns Rev mast 2 for the years 2000-2003. 


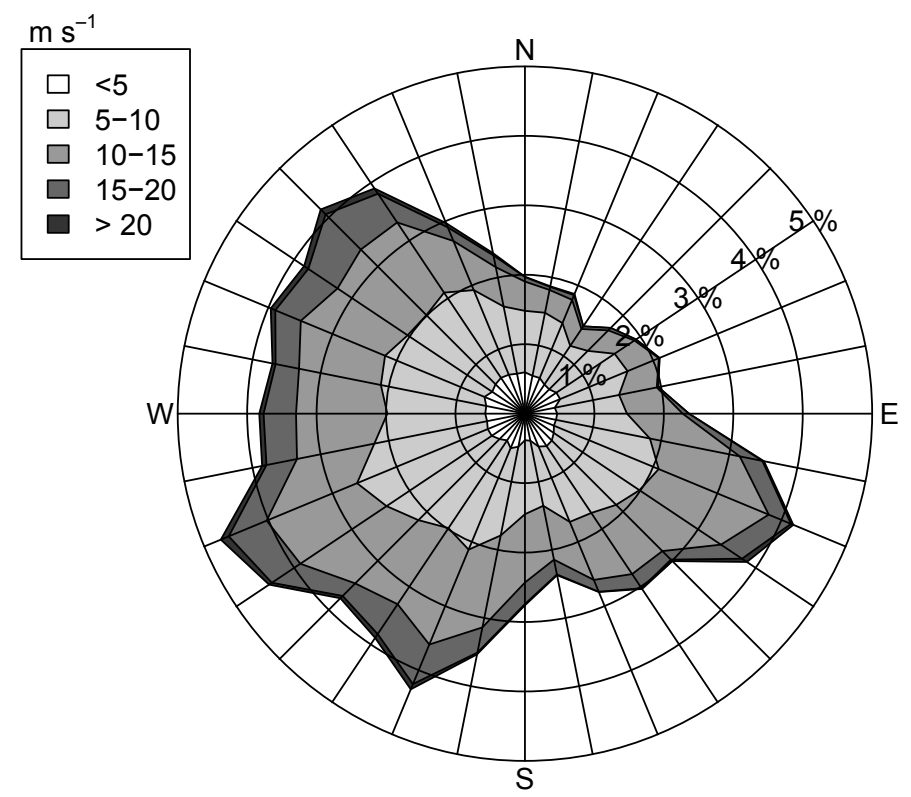

Fig. 3. Wind rose for Horns Rev mast 2 for the years 2000-2003. Angles are the direction from which the wind blows. 

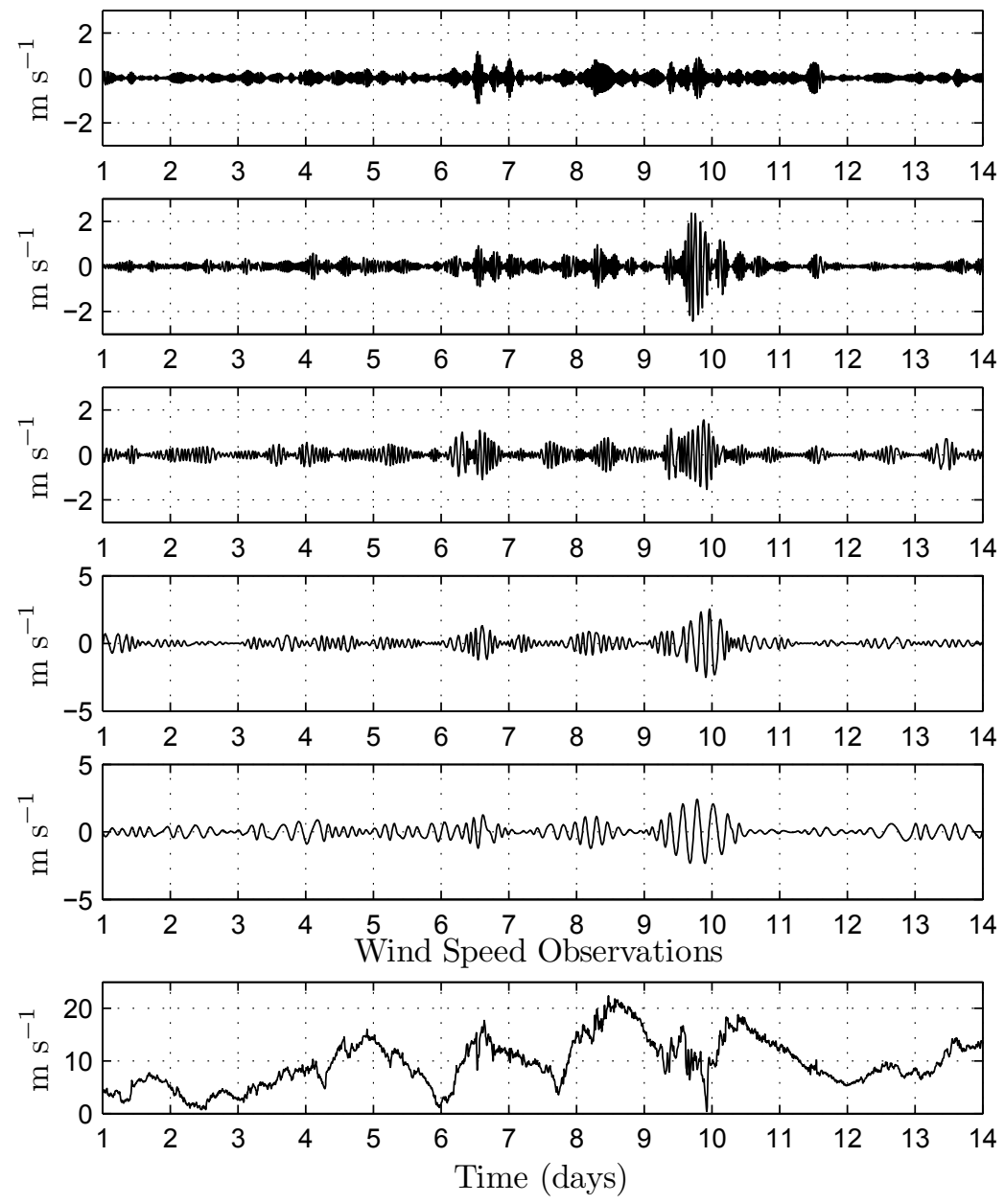

FIG. 4. The first five IMFs from the Empirical Mode Decomposition of a wind speed time series. The bottom panel shows the corresponding wind speed observations. 

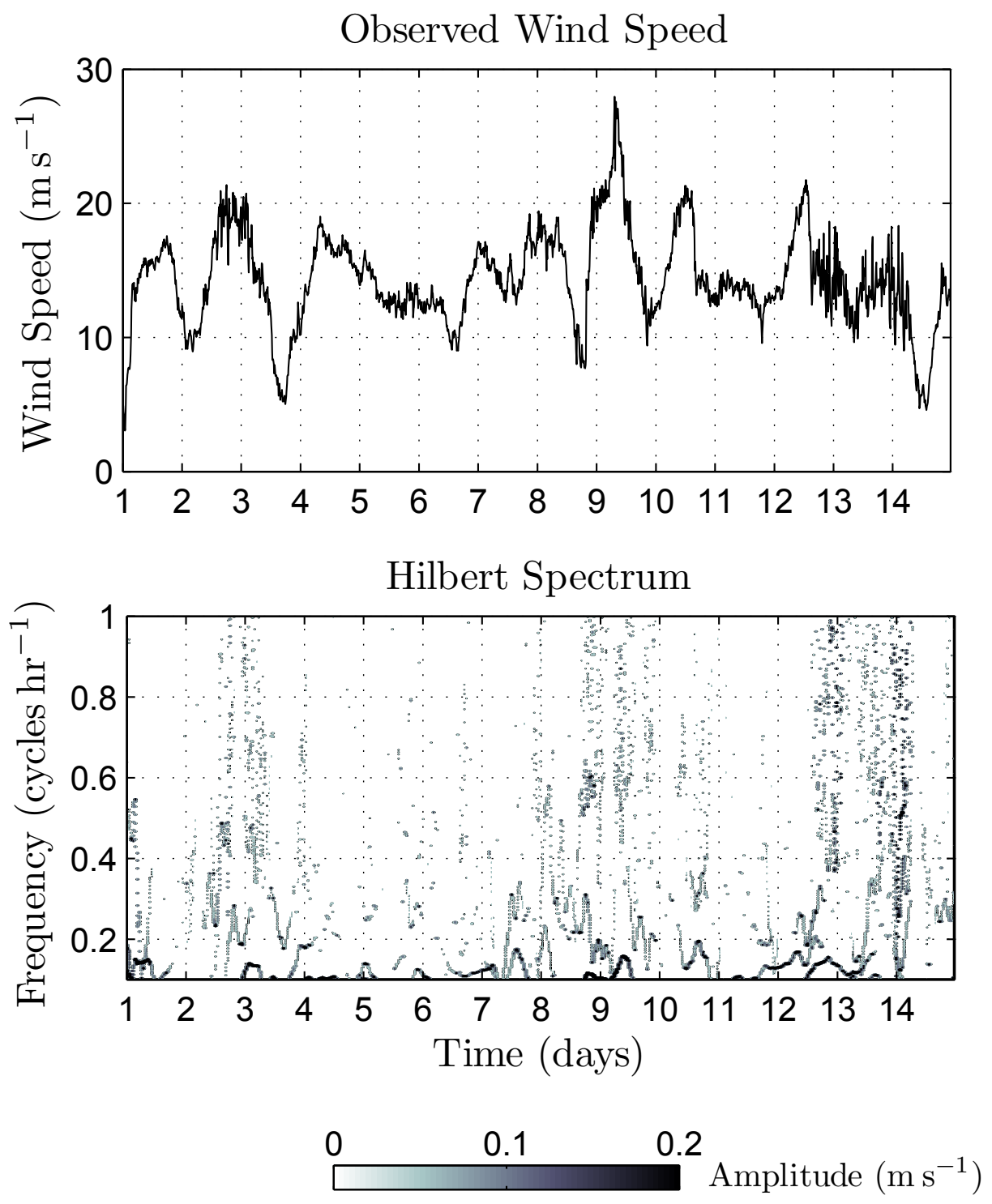

Fig. 5. Hilbert Spectrum (lower panel) of 10 minute wind observations at Horns Rev Mast 2 for a two week period in February 2000. The corresponding wind speed time series is given in the upper panel. Darker contours mean higher amplitude fluctuations, and lighter contours mean smaller amplitude fluctuations. 


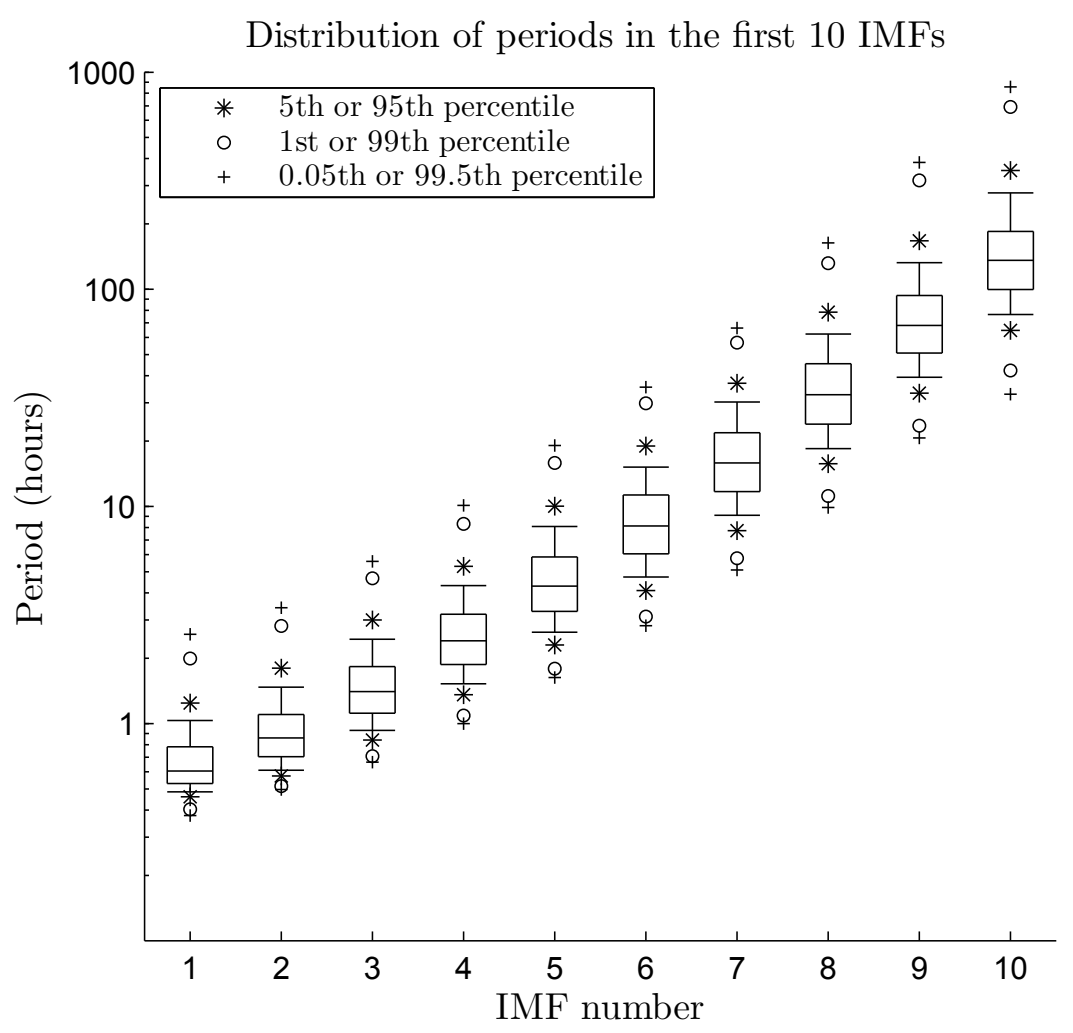

Fig. 6. Distribution of periods for the first 10 IMFs for the years 2000-2003. The whiskers show the 90th and 10th percentiles, and the symbols *, o and + show the 5th or 95th, 1st or 99th and 0.5 th or 99.5 th percentiles respectively. 


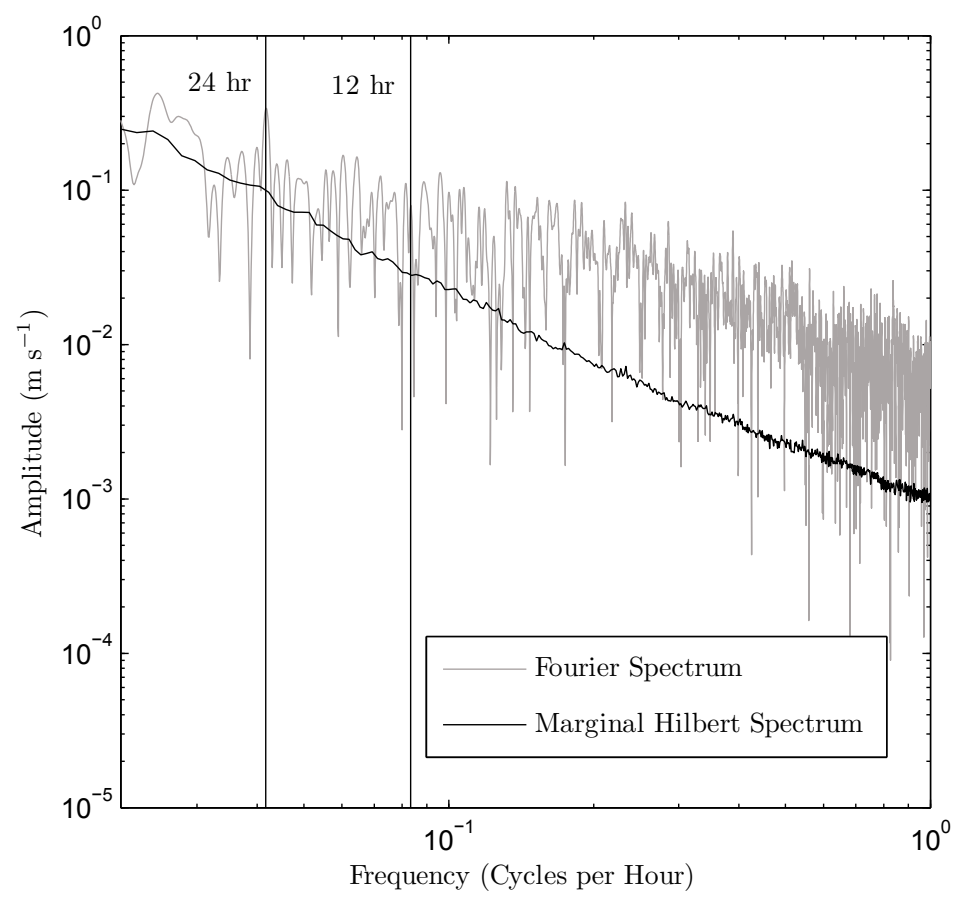

FIG. 7. Smoothed Fourier spectrum (calculated using a discrete Fourier transform and a Hanning window function) and Hilbert-Huang marginal spectrum for a 1 year time series of 10 minute wind observations 

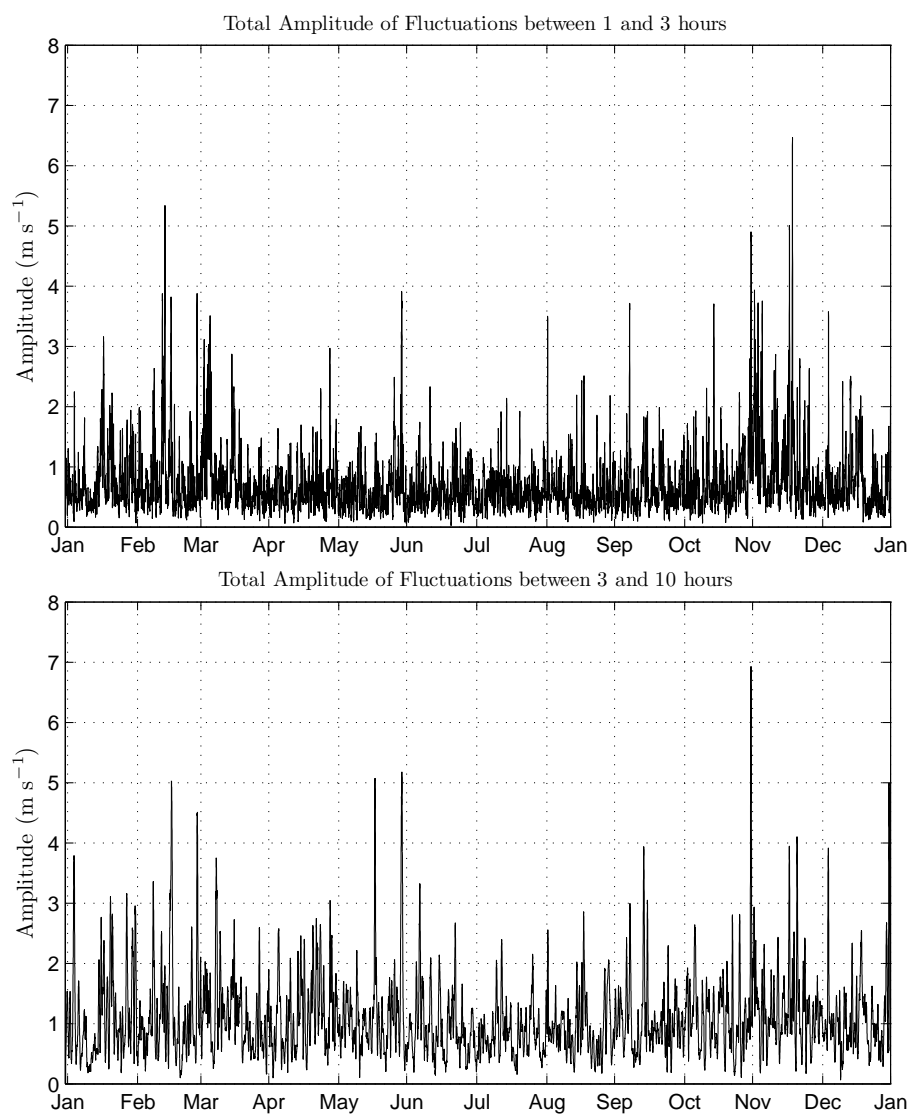

FIG. 8. Time series of total amplitude of fluctuations for periods 1 to 3 hours (upper panel) and 3 to 10 hours (lower panel), for the year 2000. The time series were smoothed using centered moving average smoothing with a window length of 3 hours (1 to 3 hour variability time series) and 10 hours (3 to 10 hour variability time series). 

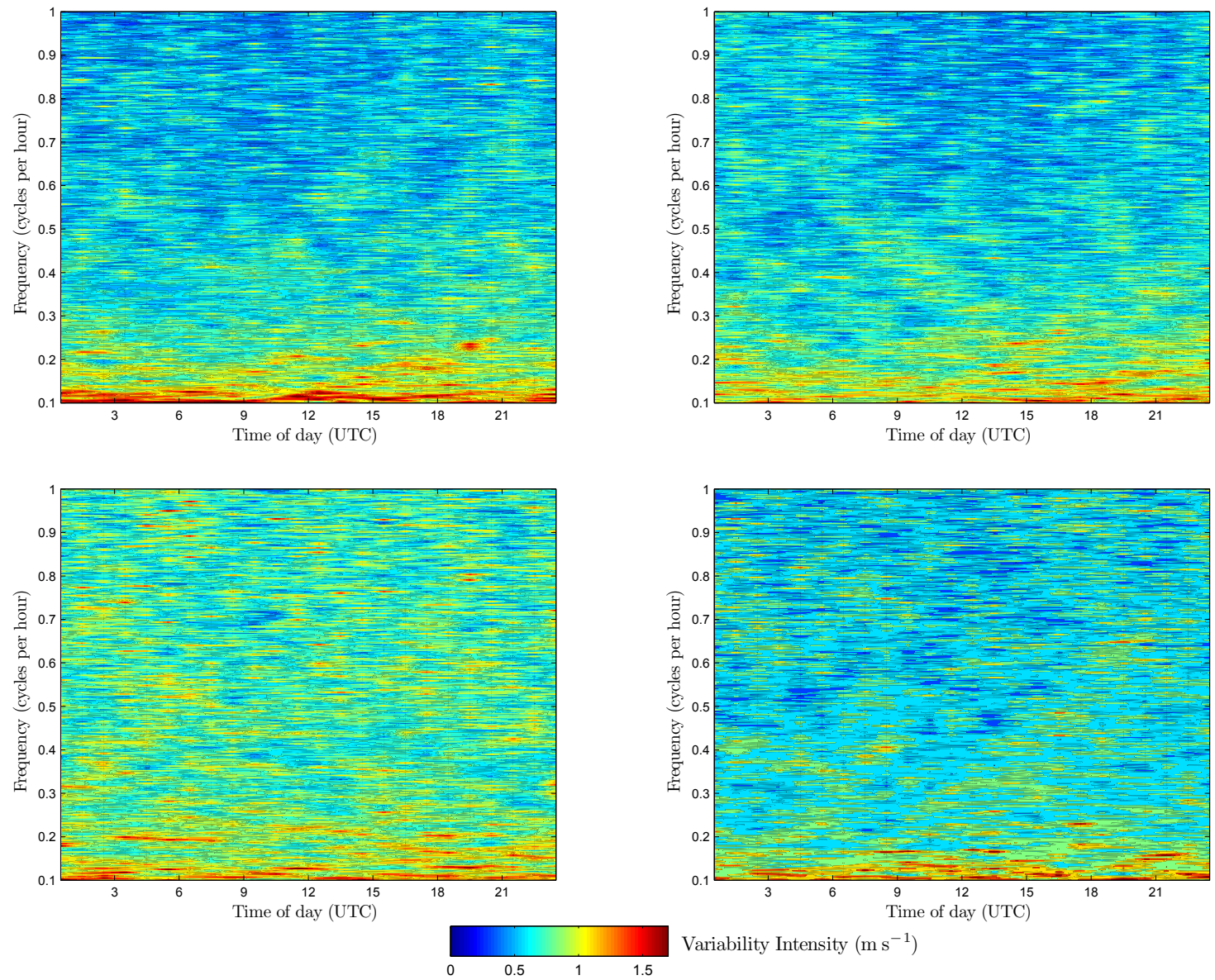

FiG. 9. Hilbert-Huang spectra, averaged for the years 2000-2003 according to time of day. Spring (top left), Summer (top right), Autumn (bottom left) and Winter (bottom right) 

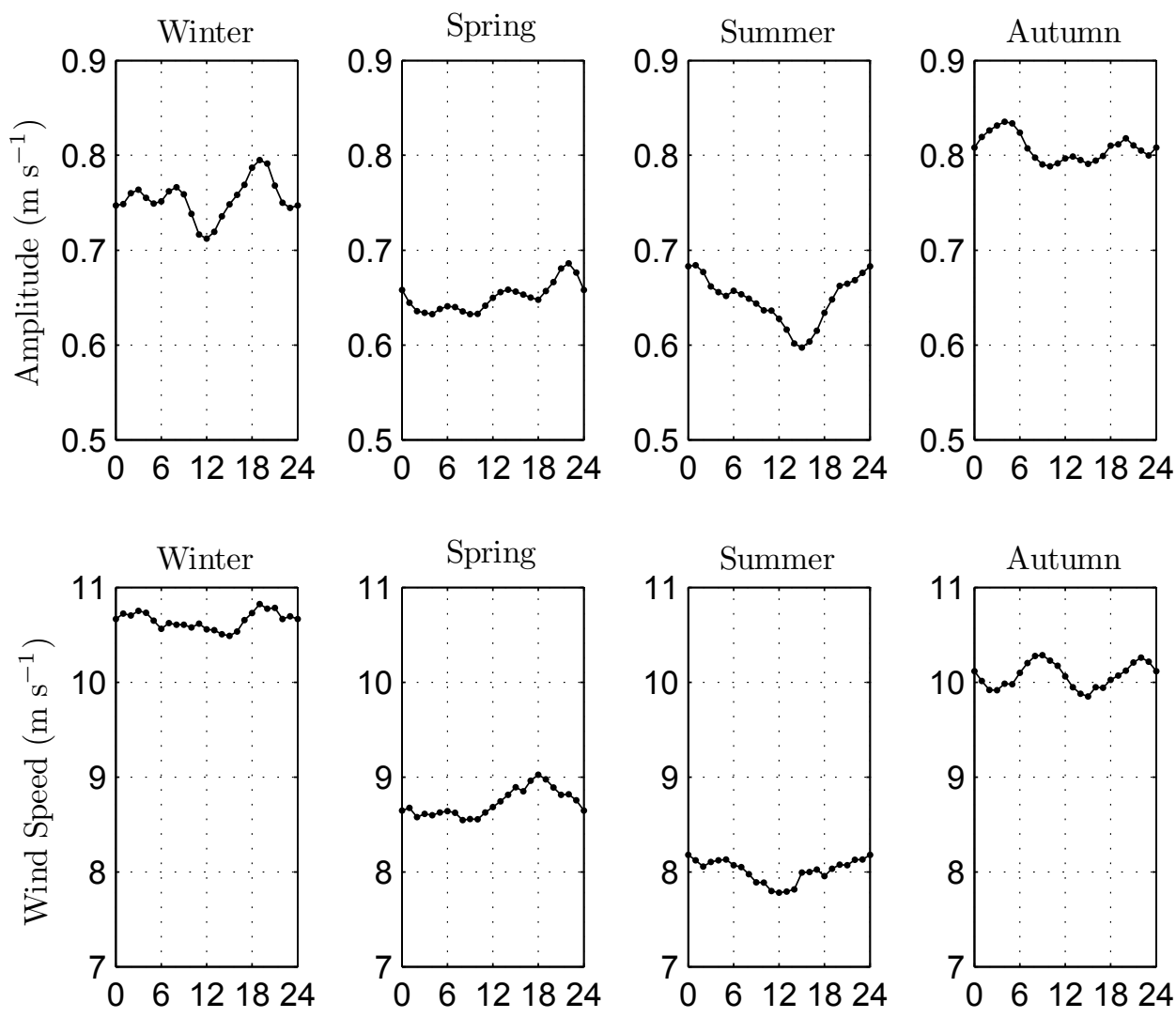

FIG. 10. Upper panel: Total amplitude of variability on scales of 1-3 hours as a function of time of day, averaged for the years 2000-2003. Lower panel: Wind speed as a function of time of day, averaged for the years 2000-2003. 


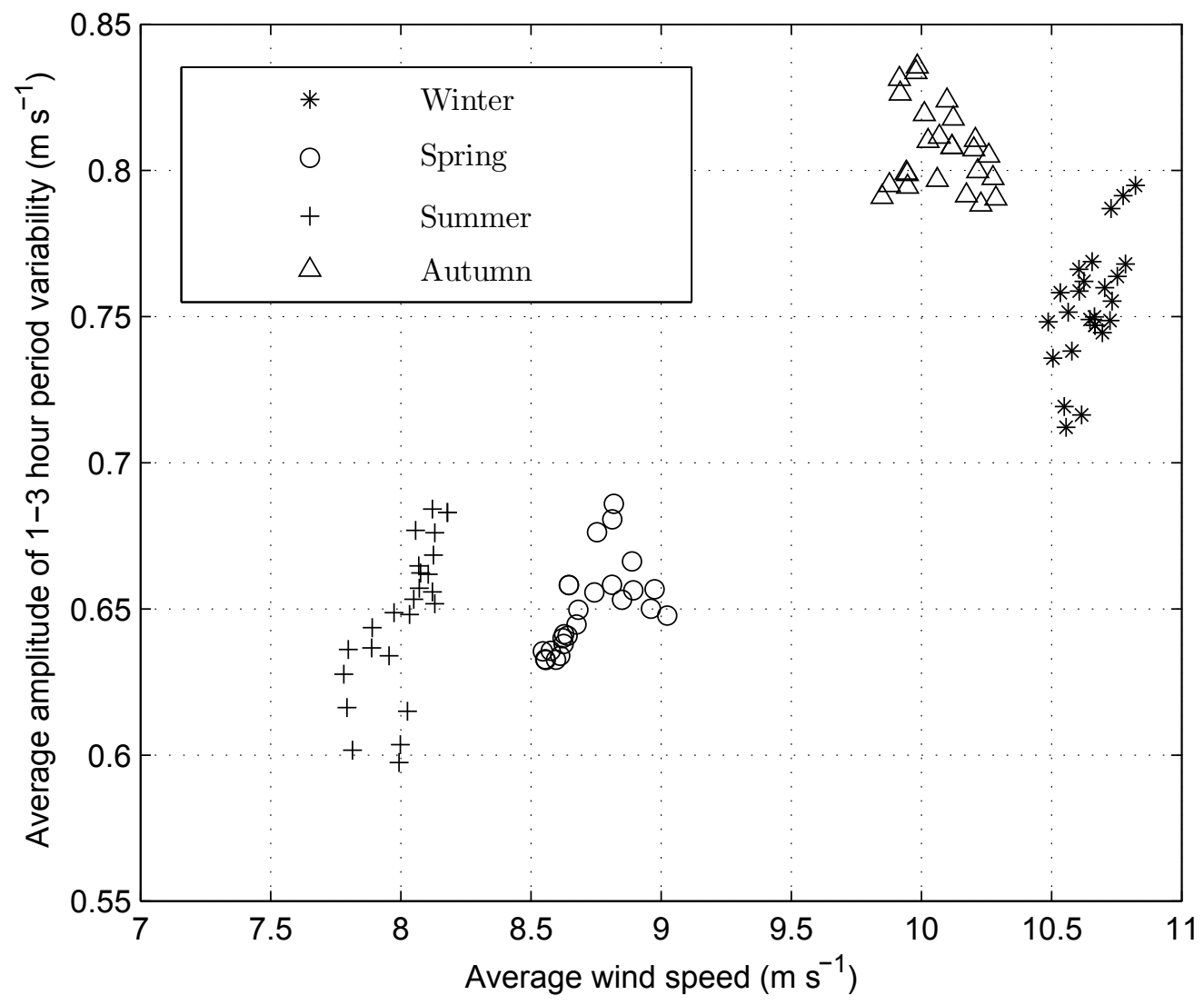

FIG. 11. The same data as in figure 10, plotted as average variability on scales of 1-3 hours against average wind speed. 